\title{
Nonlinear Tubular Organ Modeling and Analysis for Tracheal Angioedema by Swelling-Morphoelasticity
}

\author{
Kun Gou • Pak-Wing Fok • Yibin Fu
}

Received: date / Accepted: date

\begin{abstract}
This article studies one of the important human tubular organs, the trachea, under deformation caused by the disease angioedema. This pathology can suddenly increase the volume of the trachea and cause serious breathing difficulty. The swelling theory and morphoelasticity theory, which generalize classical hyperelasticity to study material deformation under internal volume change, are integrated in one single model to study tracheal angioedema. Computational modeling results from various combinations of swelling and morphoelasticity are compared to exhibit the difference and similarity of the two theories in modeling tracheal angioedema. Nonlinear behaviors of the tubular radius change are also illustrated to show how the trachea luminal size alteration depends on the swelling/growth parameters and their effect on modifying tissue stiffness. The possibility of complete tracheal channel closure is also studied to understand if it is possible for the angioedema to close the airway. This article serves as an exemplary study on nonlinear deformation behaviors of human tubular organs sharing similar structures and physiological functions.
\end{abstract}

Keywords Trachea · Angioedema $\cdot$ Swelling $\cdot$ Morphoelasticity $\cdot$ Hyperelasticity · Growth

K. Gou

Texas A\&M University-San Antonio, Department of Sciences and Mathematics, San Antonio, Texas 78224, The United States. E-mail: kun.gou@tamusa.edu

P.-W. Fok

Department of Mathematical Sciences, University of Delaware, Newark, Delaware 19716, The United States. E-mail: pakwing@udel.edu

$\mathrm{Y} . \mathrm{Fu}$

School of Computing and Mathematics, Colin Reeves Building, Keele University, Staffordshire, ST5 5BG, The United Kingdom. E-mail: y.fu@keele.ac.uk 


\section{Introduction}

The human body incorporates numerous tubular organs (TOs) responsible for transporting fluids, nutrients and waste products throughout the body. Typical TOs include blood vessels, lymphatic vessels, the colon, trachea and esophagus etc. They are mainly composed of soft tissues, and commonly exhibit nonlinear behaviors in size change [1,31, morphology [2,3], stress distribution [4] etc. This article uses the trachea under angioedema as an example to illustrate such behaviors. In particular, we model trachea angioedema (TA) using generalized hyperelasticity to see how size change and stress distribution demonstrate nonintuitive nonlinear behaviors.

The trachea is a TO closely adjacent to the esophagus. It provides a duct for air flow between the ambient environment and the two lungs. Structurally similar to many other TOs, it has multi-layers, and two distinct layers have been observed in it 5. The inner layer consists of mucous/submucous soft tissue. A longitudinal fiber family is also shown in this layer [9]. The outer layer includes numerous incomplete $\mathrm{C}$-shaped hyaline cartilage rings stacking one over another, where the unclosed part of the C-rings are just behind the esophagus [5].

TA refers to a disease under which the inner layer of the trachea accumulates fluid from blood vessel leakage through the difference of hydrostatic and osmotic pressures 10,21,22 and increases its volume rapidly 12. Such volume increment is also accompanied by mass addition such as vasoactive mediators or histamine to the tracheal tissue [11. This disease is mainly categorized into two types [11: acquired angioedema and hereditary angioedema. The first kind is caused by a deficiency of acquired c1-inhibitor c1-INH, and the second one is an autosomal dominantly inherited blood disorder [14. Regardless of which type, a rapid occurrence of volume increment on the airway soft tissue may quickly narrow the opening passage for normal breathing and induce an unexpected emergency [15, 16, 17. The pathophysiological principle of TA has been studied via lab experiments [11] or mathematical simulations [18, 19. The volume increment of the trachea is a complicated process. Some researchers applied mass conservation 23, transport theory 6], fluid mechanics 7] etc. to model such process. However, these methods are inadequate for more accurately describing the luminal area change and stress distribution from a nonlinear continuum mechanics perspective, which are key factors for evaluation of how TA may generate breathing difficulty and hurt the internal tissue.

Soft tissues exhibit behaviors including anisotropy, nonhomogeneity, strainrate dependence etc. 20]. When subject to physiological loads, soft tissues can be approximated as elastic materials and are broadly modeled by hyperelasticity [13. Presently, two theories are noticeably employed to model hyperelastic material under volume increment. The first one is the swelling theory (see e.g., 36, 37, 38, 39]). This theory assumes swelling occurs isotropically in all the directions of the material body, and the classical hyperelasticity is generalized to incorporate the swelling effect with a localized swelling parameter. 
The modified strain energy density function takes zero value on the deformed configuration after free swelling when the material body is subjected to no external constraint. The second hyperelastic theory called morphoelasticity [26] also bears the capability of modeling material volume change, and is widely applied in modeling growth of biological tissues [40,41, 42,43. The total deformation gradient tensor is decomposed to include an elastic tensor and a growth tensor. The elastic tensor is employed as the deformation gradient in the strain energy function to generate stress distribution, and its determinant is identical to one when the material is modeled by incompressibility. All the volume change caused by growth is prescribed in the growth tensor. Unlike the swelling theory, the strain energy density function is not modified in any extent. Via the growth tensor, the morphoelasticity theory is also capable of modeling anisotropic volume change.

In 24, 29, Gou and Pence modeled TA employing the swelling theory. In 24, the trachea is idealized as a closed two-layered cylinder. The top and bottom ends are taken to be force-fre $e^{i}$ and the inner and outer boundary are taken to be traction-free. Such boundary conditions assume that the deformation is merely caused by internal swelling instead of any external constraint. Angioedema generates residual stress over the tracheal body, and demonstrates high stress concentration near the interface of the inner and outer layers. In another article 29, more realistic tracheal geometries were considered that included the back-side trachealis muscle and two bronchi. Stress distribution from all levels of modeling are very similar, which justifies that idealized modeling and more practical modeling may mutually complement each other to more deeply understand geometrical change and mechanical effect brought by TA.

In this article, the swelling theory and morphoelasticity theory are integrated in one model. The new model called swelling-morphoelasticity is reflective of both swelling and morphoelasticity characteristics, and thus more flexible in modeling tissue volume-change behavior according to different compositions of swelling and growth amount. This model can also be easily reduced to either pure swelling or pure morphoelasticity. We aim at understanding the difference and similarity of the computational outcomes given by the two theories from a modeling perspective. For convenience of analysis and comparison, we idealize the trachea as a two-layered cylindrical tube following [24]. For TA, another issue deserving great attention is whether the angioedema-incurred expansion of the trachea can close the internal channel to completely prevent air flow. By intuition, large expansion may finally shrink the opening until it is totally closed. However, reality may be different especially for models using nonlinear continuum mechanics. We analyze such possibility from a perspective of mathematical rigor following the modeling part.

The structure of the article is as follows. In Section 2, we briefly introduce the generalized hyperelasticity for both swelling and morphoelasticity.

\footnotetext{
i Force free boundary conditions are different from traction-free boundary conditions. The force-free boundary condition means the integration of traction over the related boundary is annihilated.
} 
We also construct the new swelling-morphoelasticity theory and analyze its characteristics. In Sec. 3, we set up models for TA, which occurs in the inner submucous layer of the two-layered cylindrical geometry. The computational results are demonstrated in Sec. 4, where we compare modeling results from several parameter sets showing different swelling and growth amount. In Sec5 we illustrate the non-monotonic change of the luminal radius under volume increment of the trachea, and make an analysis for possibility of complete lumen closure under the swelling-morphoelasticity model. Then finally a summary discussion is given in Sec. 6 about the nonlinear TO behaviors of the trachea under angioedema.

\section{Preliminaries}

The undeformed and deformed configurations are denoted by $\Omega_{0}$ and $\Omega$, respectively. Let $\mathbf{X}$ be any generic point in $\Omega_{0}$, and it is mapped to another point $\mathbf{x}$ in $\Omega$ via a mapping $\mathbf{x}=\chi(\mathbf{X})$. The deformation gradient tensor is given by $\mathbf{F}=\partial \chi(\mathbf{X}) / \partial \mathbf{X}$. The right Cauchy-Green deformation tensor is then

$$
\mathbf{C}=\mathbf{F}^{\mathrm{T}} \mathbf{F}
$$

with the principal invariants

$$
I_{1}=\operatorname{tr} \mathbf{C}, \quad I_{2}=I_{3} \operatorname{tr}\left(\mathbf{C}^{-1}\right), \quad I_{3}=\operatorname{det} \mathbf{C} .
$$

Under any strain energy function $W$, the Cauchy stress tensor $\mathbf{T}$ is derived via

$$
\mathbf{T}=-p \mathbf{I}+2 \mathbf{F} \frac{\partial W}{\partial \mathbf{C}} \mathbf{F}^{\mathrm{T}},
$$

where $p$ is a constraint parameter and $\mathbf{I}$ is the identity tensor. $\mathbf{T}$ satisfies the equilibrium equation

$$
\operatorname{div} \mathbf{T}=\mathbf{0} .
$$

Soft tissues are usually light, so we ignore the body forces from gravity and other effects. Below we briefly review theories of swelling and morphoelasticity and form an integrated new theory based on them.

\subsection{Swelling theory}

The swelling theory is mainly applied to study fluid-filled expansion of a body. It is characteristic of 3-D isotropic volume change, and employed in areas like gel expansion and soft tissue edema [8,29]. The local swelling parameter $\nu$ depicts the ratio of the local volume in $\Omega_{0}$ and $\Omega$. For inhomogeneous swelling, $\nu$ depends on the position vector $\mathbf{X}$. In this article, for simplicity we take $\nu$ to be homogeneous and thus a constant throughout the material body. Via the deformation gradient, one has

$$
\operatorname{det} \mathbf{F}=\nu .
$$


The right Cauchy-Green deformation tensor is defined by 2.1., and its invariants are given by 2.2 . This theory can be applied in the context of both incompressibility and compressibility. In the fist case, we also call it volumespecified swelling theory. With $\nu \equiv 1$, we recover the classical incompressible hyperelastic theory.

Under a natural free swelling, i.e. boundaries of a material body are traction free, there is no elastic energy stored inside the body, and then $\mathbf{F}=\nu^{1 / 3} \mathbf{I}$. Any strain energy density function $W$ under this state arrives at a zero energy state. The energy function is generalized to reflect this result. We use neo-Hookean model as an example to show how such generalization is performed. The neoHookean model is originally given by

$$
W=\frac{\mu}{2}\left(I_{1}-3\right),
$$

where $\mu$ is the shear modulus of the material. To incorporate swelling, it is generalized to

$$
W=\frac{1}{2} \mu \nu^{q-2 / 3}\left(I_{1}-3 \nu^{2 / 3}\right),
$$

where $q$ is a parameter to show how swelling impacts on stiffness of the material, and the defaulted value $q=2 / 3$ means the swelling keeps stiffness unchanged. Other strain energy models can be generalized in a similar way to incorporate swelling.

Under a natural free swelling, the material body is stress free. The generalized relation between the Cauchy stress tensor $\mathbf{T}$ and the energy function $W$ for the volume-specified modeling is 28

$$
\mathbf{T}=-p \mathbf{I}+\frac{2}{\nu} \mathbf{F} \frac{\partial W}{\partial \mathbf{C}} \mathbf{F}^{\mathrm{T}}
$$

\subsection{Morphoelasticity}

Morphoelasticity mainly studies growth of a body through mass addition to change its volume. The growth process causes stress redistribution inside the body, and such stress and growth also demonstrate a complicated inter-connected relationship. Continuum mechanics plays a fundamental role in studying growth and its stress effect. Rodriguez et al. 25 proposed that the deformation of a body is caused by both the growth and elastic response, and they decomposed the deformation gradient $\mathbf{F}$ into two parts. One part is the growth tensor showing how mass is added into the body, and the other one is the elastic tensor, a natural response to the external mechanical stimuli and the growth to keep the body in an equilibrium state. More specifically,

$$
\mathbf{F}=\mathbf{F}_{e} \mathbf{F}_{g},
$$

where $\mathbf{F}_{e}$ is the elastic tensor and $\mathbf{F}_{g}$ is the growth tensor. The right CauchyGreen deformation tensor is defined to be on $\mathbf{F}_{e}$ only, i.e.,

$$
\mathbf{C}_{e}=\mathbf{F}_{e}^{\mathrm{T}} \mathbf{F}_{e} .
$$


One can also define the three invariants of $\mathbf{C}_{e}$ according to 2.2 .

All the volume change is incorporated in the growth tensor. The material is taken to be incompressible after growth, and then

$$
\operatorname{det} \mathbf{F}_{e}=1 \text {. }
$$

The Cauchy stress is only caused by the elastic deformation part. Denote the invariants of $\mathbf{C}_{e}$ by $I_{1}^{e}, I_{2}^{e}$ and $I_{3}^{e}$, respectively. The classical strain energy functions need not to be updated, but the invariants used in the energy functions are from $\mathbf{C}_{e}$ now. Particularly, the neo-Hookean model becomes

$$
W=\frac{\mu}{2}\left(I_{1}^{e}-3\right) .
$$

Under any strain energy function $W$, the Cauchy stress tensor is derived as

$$
\mathbf{T}=-p \mathbf{I}+2 \mathbf{F}_{e} \frac{\partial W}{\partial \mathbf{C}_{e}} \mathbf{F}_{e}^{\mathrm{T}}
$$

If $\mathbf{F}_{g}=\varepsilon \mathbf{I}$, where $\varepsilon$ is a growth parameter, the growth is isotropic. Otherwise, we call it anisotropic growth. Partial or planar isotropic growth can also be defined. Under the cylindrical coordinate system with three coordinates $(r$, $\theta, z)$, if the growth over the radial and angular directions are identical, we call it $r-\theta$ isotropic growth. Similarly, we can have $\theta-z$ or $r-z$ isotropic growth.

\subsection{Swelling-morphoelasticity}

Swelling and morphoelasticity can also be incorporated in one single model. The deformation includes both the growth part $\mathbf{F}_{g}$ and elastic part $\mathbf{F}_{e}$ as in 2.9 . In this new model, however, the volume change contribution is from both the growth and elastic parts. The growth part is for the volume change caused purely from growth, while the elastic part is responsible for the volume change caused by swelling satisfying

$$
\operatorname{det} \mathbf{F}_{e}=\nu \text {. }
$$

A deformation cartoon is shown in Fig. 1 to show such structure. The CauchyGreen tensor is identical to that defined in 2.10). The corresponding strain energy function is modified to include swelling represented by (2.14). When the neo-Hookean model is used, the updated one is

$$
W=\frac{1}{2} \mu \nu^{q-2 / 3}\left(I_{1}^{e}-3 \nu^{2 / 3}\right) .
$$

The Cauchy stress tensor is derived via

$$
\mathbf{T}=-p \mathbf{I}+\frac{2}{\nu} \mathbf{F}_{e} \frac{\partial W}{\partial \mathbf{C}_{e}} \mathbf{F}_{e}^{\mathrm{T}}
$$

We call such integration the swelling-morphoelasticity theory. This theory can easily reduce to either pure swelling hyperelastic theory via $\mathbf{F}_{g}=\mathbf{I}$ or morphoelasticity via $\nu=1$. 


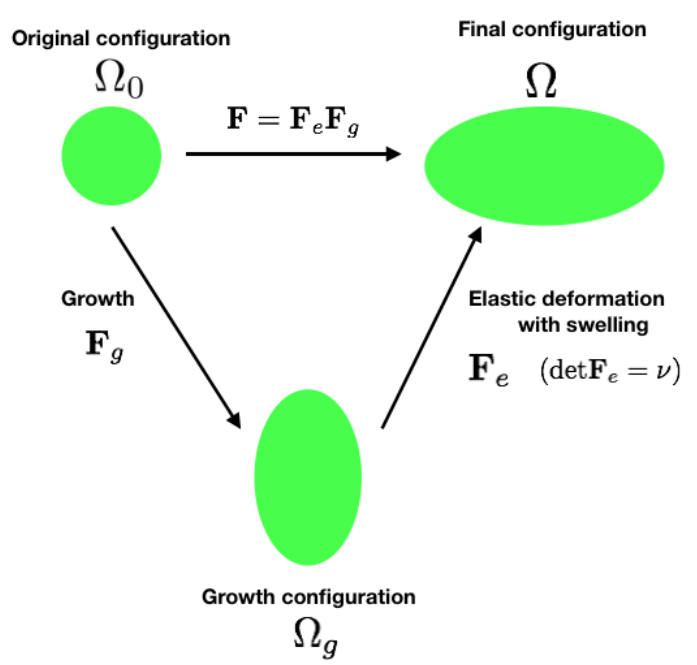

Fig. 1: Deformation cartoon for swelling-morphoelasticity. The original configuration is $\Omega_{0}$. Then after growth with the growth tensor $\mathbf{F}_{g}$, we get a visual growth configuration $\Omega_{g}$. An elastic deformation $\mathbf{F}_{e}$ is imposed on $\Omega_{g}$ to generate the final configuration $\Omega$. Swelling is also incorporated in this step, and $\operatorname{det} \mathbf{F}_{e}=\nu$. The deformation gradient $\mathbf{F}$ from $\Omega_{0}$ to $\Omega$ satisfies $\mathbf{F}=\mathbf{F}_{e} \mathbf{F}_{g}$.

\section{TA modeling via swelling-morphoelasticity}

The trachea is idealized to be a two-layered cylinder (Fig. 2). We work in a cylindrical coordinate system with the $z$-axis being the longitudinal direction of the trachea and the origin being the center of any of its horizontal cross section. The radii of the undeformed inner boundary, interface and outer boundary towards the central axis are denoted by $R_{i}, R_{m}$ and $R_{o}$, respectively. Denote the three unit basis vectors of this coordinate system by $\mathbf{e}_{R}, \mathbf{e}_{\Theta}$ and $\mathbf{e}_{Z}$ for the undeformed configuration. Any point $\mathbf{X}$ in the undeformed configuration is denoted by $(R, \Theta, Z)$. We take the same set of basis vectors for the deformed configuration due to axisymmetry of the geometry and deformation. The point $\mathbf{X}$ is mapped into another point $\mathbf{x}$ on the deformed configuration via an axisymmetric deformation function $\chi$

$$
\mathbf{x}=\chi(\mathbf{X})=r(R) \mathbf{e}_{R}+Z \lambda_{z} \mathbf{e}_{Z}
$$

where $r(R)$ is the radial function and $\lambda_{z}$ is the axial stretch parameter, respectively. The radii of the deformed inner boundary, interface and outer boundary are $r_{i}=r\left(R_{i}\right), r_{m}=r\left(R_{m}\right)$ and $r_{o}=r\left(R_{o}\right)$, respectively. The deformation gradient tensor is thus

$$
\mathbf{F}=r^{\prime}(R) \mathbf{e}_{R} \otimes \mathbf{e}_{R}+\frac{r}{R} \mathbf{e}_{\Theta} \otimes \mathbf{e}_{\Theta}+\lambda_{z} \mathbf{e}_{Z} \otimes \mathbf{e}_{Z}
$$




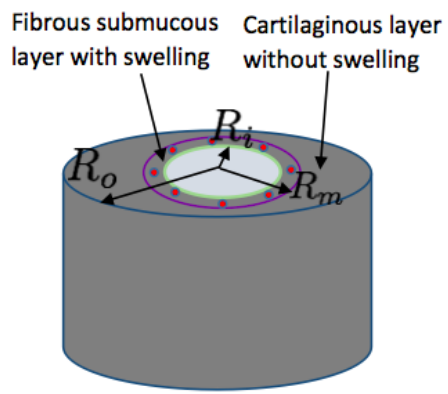

Fig. 2: An idealized two-layered trachea geometry. The inner thin layer is for submucous tissue where angioedema occurs. The red dots represent the vertically aligned collagen fibers in this layer in support of the stability of the tracheal structure. The outer thicker layer is the cartilaginous layer. This layer is mainly composed of a stack of harder cartilage rings and does not supply angioedema occurrence.

\subsection{Inner layer}

The growth tensor is taken to be in the three principal directions as

$$
\mathbf{F}_{g}=g_{r} \mathbf{e}_{R} \otimes \mathbf{e}_{R}+g_{\theta} \mathbf{e}_{\Theta} \otimes \mathbf{e}_{\Theta}+g_{z} \mathbf{e}_{Z} \otimes \mathbf{e}_{Z},
$$

where $g_{r}, g_{\theta}$ and $g_{z}$ are growth parameters in the three principal directions, respectively.

By 2.9, 2.14 and 3.2

$$
\mathbf{F}_{e}=\frac{1}{\alpha} \mathbf{e}_{R} \otimes \mathbf{e}_{R}+\frac{\nu g_{z}}{\lambda_{z}} \alpha \mathbf{e}_{\Theta} \otimes \mathbf{e}_{\Theta}+\frac{\lambda_{z}}{g_{z}} \mathbf{e}_{Z} \otimes \mathbf{e}_{Z},
$$

where,

$$
\alpha=\frac{g_{r}}{r^{\prime}}, \quad \frac{\nu g_{z}}{\lambda_{z}} g_{\theta} \alpha=\frac{r}{R} .
$$

It then follows from 2.14 that

$$
\left(r^{2}\right)^{\prime}=\frac{2 \nu g_{r} g_{\theta} g_{z} R}{\lambda_{z}}
$$

and so

$$
r=\left[\int_{R_{i}}^{R} \frac{2 \nu g_{r} g_{\theta} g_{z} R}{\lambda_{z}} \mathrm{~d} R+r_{i}^{2}\right]^{1 / 2}, \quad R_{i} \leq R \leq R_{m}
$$

where $r_{i}=r\left(R_{i}\right)$. The parameters such as $\nu$ and $g_{r}$ may be functions of $R$ instead of constants, and thus (3.7) can not be further simplified at this step. By (3.7), we can also derive the relation between $r_{m}$ and $r_{i}$

$$
r_{m}^{2}=r_{i}^{2}+\int_{R_{i}}^{R_{m}} \frac{2 \nu g_{r} g_{\theta} g_{z} R}{\lambda_{z}} \mathrm{~d} R,
$$


which further generates another identical formula to 3.7

$$
r=\left[r_{m}^{2}-\int_{R}^{R_{m}} \frac{2 \nu g_{r} g_{\theta} g_{z} R}{\lambda_{z}} \mathrm{~d} R\right]^{1 / 2}, \quad R_{i} \leq R \leq R_{m}
$$

The strain energy density function of the inner layer is taken to be the addition of the generalized neo-Hookean strain energy and longitudinal fiber energy as

$$
W_{i}=\underbrace{\frac{\mu_{i} \nu^{q-2 / 3}}{2}\left(I_{1}-3 \nu^{2 / 3}\right)}_{\text {neo-Hookean energy }}+\underbrace{\frac{\gamma}{2}\left(I_{4}-1\right)^{2}}_{\text {fiber energy }},
$$

where $\mu_{i}$ is the shear modulus of the inner submucous layer, $\gamma$ is the fiber elastic modulus, and $I_{1}$ is the first variant of $\mathbf{C}_{e}$. In $3.10, I_{4}$ is defined as a qseudo-invariant as

$$
I_{4}=\mathbf{N}_{f i b} \cdot \mathbf{C}_{e} \mathbf{N}_{f i b},
$$

where $\mathbf{N}_{f i b}=\mathbf{e}_{Z}$ is the unit direction of the longitudinal fibers embedded in the submucous layer of the trachea. By 2.16), the Cauchy stress tensor is

$$
\mathbf{T}^{i}=-p_{i} \mathbf{I}+\mu_{i} \nu^{q-5 / 3} \mathbf{B}_{e}+\frac{2 \gamma}{\nu}\left(I_{4}-1\right) \mathbf{E}_{e} \mathbf{e}_{Z} \otimes \mathbf{E}_{e} \mathbf{e}_{Z}
$$

where $\mathbf{B}_{e}=\mathbf{F}_{\mathbf{e}} \mathbf{F}_{\mathbf{e}}^{\mathrm{T}}$ is the left Cauchy-Green tensor, and $p_{i}$ is the incompressibility constraint parameter dependent on $R$. Detailed computation gives the Cauchy stress in its component-wise form to be

$$
\begin{aligned}
& T_{r r}^{i}=-p_{i}+\frac{\mu_{i} \nu^{q-5 / 3}}{\alpha^{2}}, \quad R_{i} \leq R \leq R_{m}, \\
& T_{\theta \theta}^{i}=-p_{i}+\frac{\mu_{i} g_{z}^{2} \nu^{q+1 / 3} \alpha^{2}}{\lambda_{z}^{2}}, \quad R_{i} \leq R \leq R_{m}, \\
& T_{z z}^{i}=-p_{i}+\frac{\mu_{i} \lambda_{z}^{2} \nu^{q-5 / 3}}{g_{z}^{2}}+\frac{2 \gamma \lambda_{z}^{2}}{\nu g_{z}^{2}}\left(\frac{\lambda_{z}^{2}}{g_{z}^{2}}-1\right), \quad R_{i} \leq R \leq R_{m} .
\end{aligned}
$$

The three equilibrium equations in 2.4 for this axisymmetric model reduces to only one equation in the radial direction

$$
\frac{\partial T_{r r}^{i}}{\partial r}+\frac{1}{r}\left(T_{r r}^{i}-T_{\theta \theta}^{i}\right)=0
$$

By (3.13), 3.14 and (3.16), one has

$$
\begin{aligned}
& p_{i}(R)=p_{i}\left(R_{i}\right)+ \\
& \mu_{i} \int_{R_{i}}^{R} \nu^{q-5 / 3}\left(\frac{(q-5 / 3) \nu^{-1}}{\alpha^{2}} \frac{\mathrm{d} \nu}{\mathrm{d} R}-2 \alpha^{-3} \frac{\mathrm{d} \alpha}{\mathrm{d} R}+\frac{1}{r}\left(\frac{1}{\alpha^{2}}-\frac{g_{z}^{2} \nu^{2} \alpha^{2}}{\lambda_{z}^{2}}\right) \frac{\mathrm{d} r}{\mathrm{~d} R}\right) \mathrm{d} R .
\end{aligned}
$$


3.2 Outer layer

There is no growth or swelling in the outer cartilaginous layer. Thus $\mathbf{F}_{g}=\mathbf{I}$ and $\nu=1$. By the incompressibility constraint $\operatorname{det} \mathbf{F}=1$, via 3.2 .

$$
\frac{r^{\prime} r \lambda_{z}}{R}=1
$$

Integration of 3.18 from $R$ to $R_{o}$ gives

$$
r=\sqrt{r_{o}^{2}-\frac{1}{\lambda_{z}}\left(R_{o}^{2}-R^{2}\right)}, \quad R_{m} \leq R \leq R_{o} .
$$

By $(3.19)$, we can obtain the relation between $r_{m}$ and $r_{o}$ as

$$
r_{m}^{2}=r_{o}^{2}-\frac{1}{\lambda_{z}}\left(R_{o}^{2}-R_{m}^{2}\right)
$$

which gives another formula for $r$ as

$$
r=\sqrt{r_{m}^{2}+\frac{1}{\lambda_{z}}\left(R^{2}-R_{m}^{2}\right)}, \quad R_{m} \leq R \leq R_{o}
$$

The strain energy density function is taken to be the original neo-Hookean model

$$
W_{o}=\frac{\mu_{o}}{2}\left(I_{1}-3\right),
$$

where $\mu_{o}$ is the shear modulus of the outer cartilaginous layer. The Cauchy stress tensor $\mathbf{T}^{o}$ for this layer is derived via $W_{o}$ as

$$
\mathbf{T}^{o}=-p_{o} \mathbf{I}+2 \mathbf{F} \frac{\partial W_{o}}{\partial \mathbf{C}} \mathbf{F}^{\mathrm{T}}=-p_{o} \mathbf{I}+\mu_{o} \mathbf{B},
$$

where $\mathbf{B}=\mathbf{F F}^{\mathrm{T}}$ is the left Cauchy-Green tensor for the outer layer, and $p_{o}$ is a constraint parameter for this layer. Detailed computation gives the Cauchy stress in its component-wise form to be

$$
\begin{aligned}
& T_{r r}^{o}=-p_{o}+\mu_{o}\left(r^{\prime}\right)^{2}, \quad R_{m} \leq R \leq R_{o} \\
& T_{\theta \theta}^{o}=-p_{o}+\mu_{o} r^{2} / R^{2}, \quad R_{m} \leq R \leq R_{o} \\
& T_{z z}^{o}=-p_{o}+\mu_{o} \lambda_{z}^{2}, \quad R_{m} \leq R \leq R_{o}
\end{aligned}
$$

As in 3.16 , we get one equilibrium equation in the radial direction

$$
\frac{\partial T_{r r}^{o}}{\partial r}+\frac{1}{r}\left(T_{r r}^{o}-T_{\theta \theta}^{o}\right)=0 .
$$

By virtue of (3.24, 3.25) and 3.27, we get

$$
p_{o}(R)=p_{o}\left(R_{o}\right)-\mu_{o} \int_{R}^{R_{o}} r^{\prime}\left(2 r^{\prime \prime}+r^{-1}\left(\left(r^{\prime}\right)^{2}-\frac{r^{2}}{R^{2}}\right)\right) \mathrm{d} R .
$$


3.3 Boundary conditions and solution procedure

The inner boundary of the tracheal tube is subject to aerial flow to and from the two lungs. The pressure is close to the atmospheric pressure which can be balanced by the pressure inside the tracheal tissue. We thus ignore the effect on the inner boundary of the trachea exerted by the air flow inside the lumen, and take the inner boundary of the trachea tube to be traction free. The outer boundary of the trachea tube is surrounded by light soft tissue, and taken to be traction free. We also take a traction-free inner and outer boundary conditions giving

$$
\begin{cases}T_{r r}^{i}\left(r_{i}\right)=0 & \text { (traction-free inner boundary), } \\ T_{r r}^{o}\left(r_{o}\right)=0 & \text { (traction-free outer boundary). }\end{cases}
$$

By 3.13 and 3.291 , we get

$$
p_{i}\left(R_{i}\right)=\left.\frac{\mu_{i} \nu^{q-5 / 3}}{\alpha^{2}}\right|_{R=R_{i}},
$$

and by $(3.24)$ and $(3.29)_{2}$, we have

$$
p_{o}\left(R_{o}\right)=\left.\mu_{o}\left(r^{\prime}\right)^{2}\right|_{R=R_{o}} .
$$

At the interface, the traction is taken to be continuous, which gives

$$
T_{r r}^{i}\left(r_{m}\right)=T_{r r}^{o}\left(r_{m}\right) \quad \text { (traction continuous at the interface), }
$$

or by 3.13 and 3.24

$$
-p_{i}\left(R_{m}\right)+\left.\frac{\mu_{i} \nu^{q-5 / 3}}{\alpha^{2}}\right|_{R=R_{m}}=-p_{o}\left(R_{m}\right)+\left.\mu_{o}\left(r^{\prime}\right)^{2}\right|_{R=R_{m}} .
$$

The radial function $r(R)$ is also continuous throughout the interface in the domain $\left[R_{i}, R_{o}\right]$ automatically by our formulation. Namely, $r(R)$ in $(3.9)$ for the inner submucous layer and $r(R)$ in 3.21 for the outer cartilaginous layer are equal when both estimated at $R=R_{m}$. Using $(3.9)$ and $(3.21),(3.33)$ is an equation for the unknown $r_{m}$. This nonlinear equation can be solved by numerical techniques. With the value of $r_{m}$, we can obtain the Cauchy stress distribution $\mathbf{T}$ and radial function $r(R)$ for the two layers.

\section{Modeling results and comparison}

The radius values for $R_{i}, R_{m}$ and $R_{o}$ are taken from 24,29 as shown in Table 1 The material stiffness values are illustrated in Table 2 ii We use the defaulted value $q=2 / 3$ for the computation in this section, i.e., the volume increment shall not change the stiffness of the tissue. The product of all growth and swelling parameters $\nu, g_{r}, g_{\theta}$ and $g_{z}$ is fixed for convenience of comparison.

\footnotetext{
ii These values were calculated from experimental data via justified mathematical formulas. See 24].
} 


\begin{tabular}{|lcccc|}
\hline \multicolumn{6}{c|}{ Radius values (unit:mm) } \\
\hline & Notation & Inner boundary & Interface & Outer boundary \\
\hline Original size: & $R$ & $R_{i}=8.85$ & $R_{m}=9.15$ & $R_{o}=11.45$ \\
\hline After deformation: & $r$ & $r_{i}$ & $r_{m}$ & $r_{o}$ \\
\hline
\end{tabular}

Table 1: Radius values before and after deformation caused by angioedema. The radius values before deformation for the inner boundary, interface and outer boundary are taken from 24,29$]$. The corresponding boundary radii after deformation are unknowns and their values are calculated from the present model.

\begin{tabular}{|ccc|}
\hline \multicolumn{3}{|c|}{ Tracheal stiffness parameters (unit: MPa) } \\
\hline Inner layer shear modulus & Outer layer shear modulus & Fiber stiffness \\
\hline$\mu_{i}=0.0429$ & $\mu_{o}=0.58$ & $\gamma=0.0429$ \\
\hline
\end{tabular}

Table 2: Tracheal tissue stiffness values used in the model. These value are kept to be identical to those used in 2429 for consistency.

This makes the total volume change under the collective effect of growth and swelling of the submucous layer after TA identical. The product $\nu g_{r} g_{\theta} g_{z}=4$. Because the swelling theory is for volume change in $3-\mathrm{D}$, first we consider 3-D isotropic growth in the swelling-morphoelasticity model and then 2-D isotropic growth.

\subsection{3-D isotropic growth}

Isotropic growth means growth in the three principal directions $\mathbf{e}_{r}, \mathbf{e}_{\theta}$ and $\mathbf{e}_{z}$ are identical, i.e., $g_{1}=g_{2}=g_{3}=g$, where $g$ is a constant. Here we take $g^{3} \nu=4$. We use four sets of parameters as a demonstration:

$$
\begin{aligned}
& S_{1}:\{g=1, \nu=4\}, \quad S_{2}:\{g=\sqrt[3]{4 / 3}, \nu=3\} \\
& S_{3}:\{g=\sqrt[3]{2}, \nu=2\}, \quad S_{4}:\{g=\sqrt[3]{4}, \nu=1\}
\end{aligned}
$$

Set $S_{1}$ shows only swelling without growth $(g=1)$, and Set $S_{4}$ shows only growth without swelling $(\nu=1)$. They are the two extreme ends of the swelling-morphoelasticity modeling. The other two sets are mix of both swelling and growth.

Figure 3 illustrates the results under an axial stretch $\lambda_{z}=1$. First we give a summary for the general patterns of all curves. Due to the volume increment of the inner submucous layer from TA, Figure 3a shows that the inner radius $r_{i}$ is reduced and the outer radius $r_{o}$ is increased for the parameter set choice. We also remark that in this panel the curves over the inner layer or outer layer are almost straight, but they are nonlinear curves. Figure 3b shows that the radial stress $T_{r r}$ is compressive, and reaches its maximum at the interface, which makes the tissue more vulnerable at this location. The curves also show cusps indicating non-smoothness at the interface because only traction continuity condition is prescribed at this location. Figures $3 \mathrm{c}$ and $3 \mathrm{~d}$ for $T_{\theta \theta}$ and $T_{z z}$ vs. 


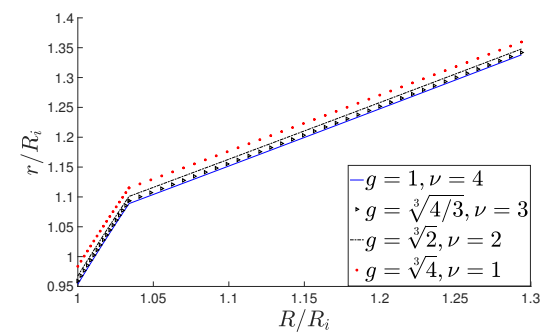

(a) $r / R_{i}$ vs. $R / R_{i}$

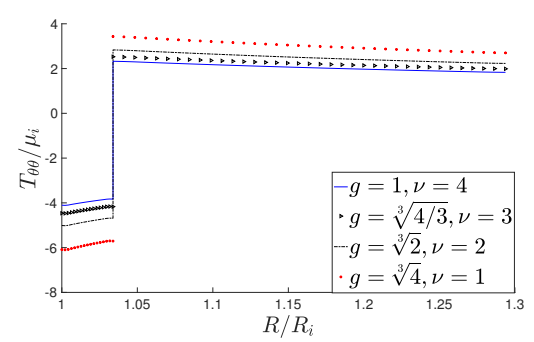

(c) $T_{\theta \theta}$ vs. $R / R_{i}$

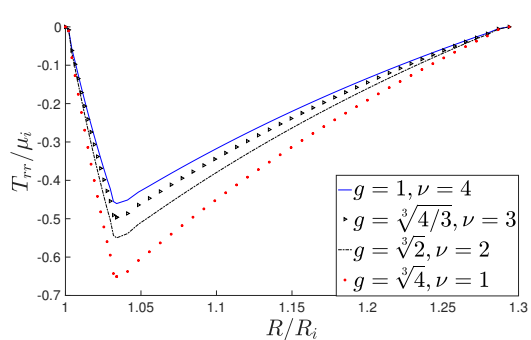

(b) $T_{r r}$ vs. $R / R_{i}$

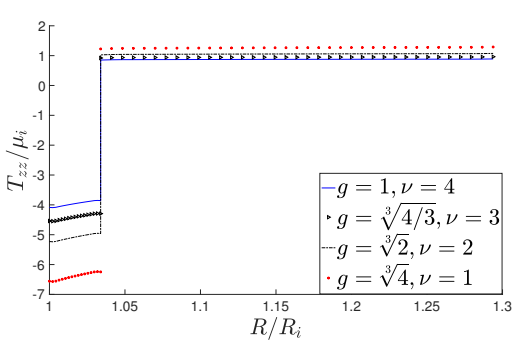

(d) $T_{z z}$ vs. $R / R_{i}$

Fig. 3: Radius change and stress distribution as functions of $R$ for various combinations of swelling and 3-D isotropic growth parameters $\nu, g_{r}, g_{\theta}$, and $g_{z}$ satisfying $g_{r}=g_{\theta}=g_{z}=g$. Here $R$ is normalized by $R_{i}$, and stress is normalized by $\mu_{i}$. The product of all growth and swelling parameters is kept to be a constant 4, i.e., $g^{3} \nu=4$. There is no stretch in the axial direction $\left(\lambda_{z}=1\right)$, and the defaulted value $q=2 / 3$ is used. The plots illustrate that increased $g$ or decreased $\nu$ generates increased $r_{i}$ (decreased trachea opening constriction) and increased stress magnitude at each fixed $R$.

$R$, respectively, show compressive stress in the inner layer and tensile stress in the outer layer, and discontinuities are generated at the interface.

Then we compare the curves in each subfigure for the four sets $S 1-S 4$. Figure $3 \mathrm{a}$ shows that Set $S_{1}$ for pure swelling gives the lowest $r$ vs. $R$ curve, while Set $S_{4}$ gives the highest, or more generally, more growth or less swelling makes the curve higher. Therefore, under the same volume change, modeling TA using more swelling produces more inner opening shrinkage. In Figures $3 \mathrm{~b}$ $3 \mathrm{~d}$, at a fixed $R$ location Set $S_{1}$ generates the smallest stress magnitude and Set $S_{4}$ generates the largest, or more generally, more growth generates larger stress magnitude. One possible explanation for this phenomenon is that more growth makes the trachea expand more outward, and thus the inner submucous layer pushes the outer cartilaginous layer more outward resulting in bigger stress production. More growth hence may more seriously hurt the tracheal tissue bearing stronger stress.

Computation for $\lambda_{z}=1.3$ is also taken. For each set of parameters, the inner opening is more constrained, and smaller stress intensity is produced. 


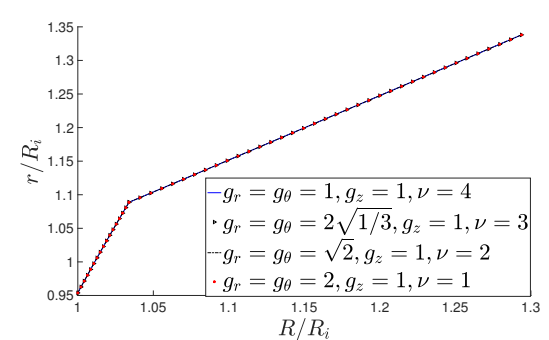

(a) $r / R_{i}$ vs. $R / R_{i}$

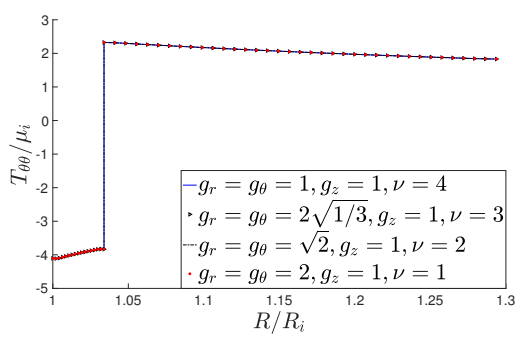

(c) $T_{\theta \theta}$ vs. $R / R_{i}$

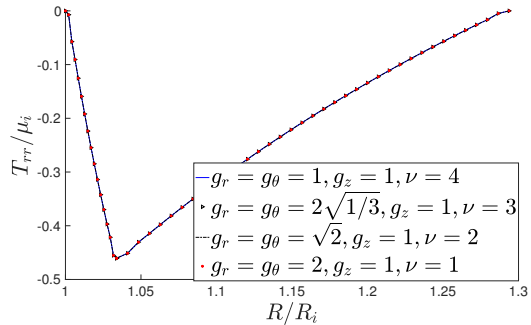

(b) $T_{r r}$ vs. $R / R_{i}$

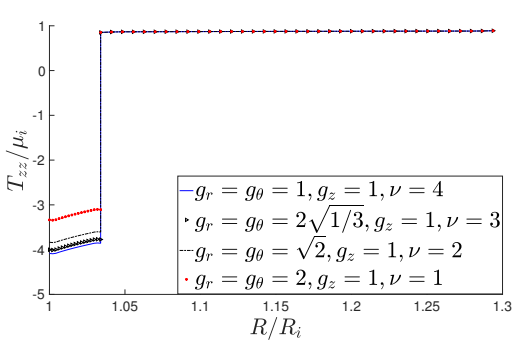

(d) $T_{z z}$ vs. $R / R_{i}$

Fig. 4: Radius change and stress distribution of various combinations of swelling and 2-D isotropic growth $\left(g_{r}=g_{\theta}, g_{z}=1\right)$, where the growth is constrained to be on the radial and angular directions. The product of all growth and swelling parameters is kept to be a constant 4, i.e., $g_{r} g_{\theta} g_{z} \nu=4$. Here $\lambda_{z}=1$ and $q=2 / 3$. The graphs show that the four sets generate the same results for radius change and $T_{r r}$ and $T_{\theta \theta}$ distributions, and $T_{z z}$ is identical only in the outer layer.

However, the results show no different pattern compared with Fig. 3 and the graph is thus skipped.

\subsection{2-D isotropic growth}

We study how the results will differ when the isotropic growth is constrained in 2-D. Three types are studied: isotropic $r-\theta$ growth, isotropic $r$ - $z$ growth, Isotropic $\theta-z$ growth.

\subsubsection{Isotropic $r-\theta$ growth}

We consider planar isotropic growth over the radial and angular directions. Here we take $g_{r}=g_{\theta}, g_{z}=1, g_{r}^{2} \nu=4$, and $\lambda_{z}=1$. Four sets of parameters 
are used as follows:

$$
\begin{aligned}
& S_{5}:\left\{g_{r}=g_{\theta}=1, g_{z}=1, \nu=4\right\}, \quad S_{6}:\left\{g_{r}=g_{\theta}=2 \sqrt{1 / 3}, g_{z}=1, \nu=3\right\}, \\
& S_{7}:\left\{g_{r}=g_{\theta}=\sqrt{2}, g_{z}=1, \nu=2\right\}, \quad S_{8}:\left\{g_{r}=g_{\theta}=2, g_{z}=1, \nu=1\right\} .
\end{aligned}
$$

The general pattern of the results for these four parameter sets shown in Fig. 4 are similar to that in Fig. 3 . For all four sets, Figure 4 demonstrates identical curves for $r(R), T_{r r}(R)$ or $T_{\theta \theta}(R)$. For the axial stress $T_{z z}$, all four curves are the same over the outer cartilaginous layer and disagree over the inner submucous layer (Set 8 for pure growth with $\nu=1$ generates the least $T_{z z}$ intensity over the inner layer). Therefore, in the swelling-morphoelasticity model, computational results are identical regardless of how swelling and growth are mixed. Nonzero $T_{z z}$ values also suggest that planar $r-\theta$ growth is not completely constrained in the $r-\theta$ plane, and also generates stress effect in the axial direction outside of the $r-\theta$ plane.

In order to test how different axial stretch can affect the deformation, we also used $\lambda_{z}=1.3$ in the computation for the four sets $S 5$ to $S 8$. The same pattern is maintained as in Fig. 4, and hence the graphs are not displayed here for the sake of brevity. It is also found that large $\lambda_{z}$ may generate all tensile stress over the two layers instead of being compressive over the inner layer and tensile over the outer layer for smaller $\lambda_{z}$. Larger $\lambda_{z}$ also produces smaller $r_{i}$ giving more constrained inner opening and causing more difficulty for breathing.

\subsubsection{Isotropic $r-z$ and $\theta-z$ growth}

The computational results of isotropic $r-z$ or $\theta-z$ growth do not show a nice overlap for different combinations of swelling and growth as those for isotropic $r-\theta$ growth. Generally the plots are similar to Fig. 3 and therefore not shown here for the sake of brevity.

\section{Luminal shrinkage}

When the trachea is under angioedema, the volume of the inner layer expands. Quantitative and qualitative understanding of the expansion is necessary for accurate description of severity of the disease. Based on the model setup in Sec. 3. we graphically obtain relations between inner radius change and volume increment amount. Then we study when it is possible for the angioedema to totally close the luminal area to obstruct air flow.

\subsection{Relation of $r_{i}$ and volume increment}

The idealized modeling for the tracheal geometry allows quantifying the luminal area by the deformed inner radius $r_{i}$. We study the relation between $r_{i}$ 


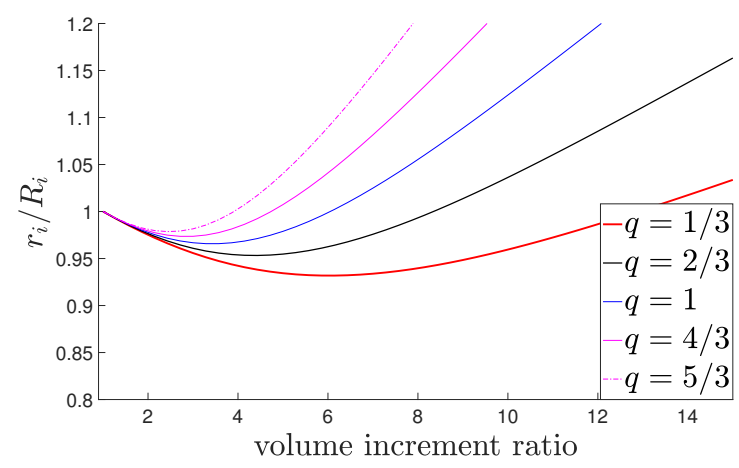

Fig. 5: Graph of how $r_{i}$ (normalized by $R_{i}$ ) changes based on total volume increment for various $q$ values indicating how volume increment alters stiffness of the material. The label for the horizontal axis "volume increment ratio" ( vir) means the pointwise volume ratio after and before angioedema satisfying $v i r=\operatorname{det} \mathbf{F}=\operatorname{det} \mathbf{F}_{e} \operatorname{det} \mathbf{F}_{g}$. Isotropic $r-\theta$ growth is used in the swelling-morphoelasticity model with growth parameters satisfying $g_{z}=1$ and $g_{r}=g_{\theta}$ For each value of vir, the contributions from swelling (with swelling parameter $\nu$ ) and total growth are the same, i.e., $\nu=\sqrt{v i r}$ and $g_{r} g_{\theta}=\sqrt{v i r}$. Each curve is non-monotonic. The function $r_{i}$ (vir) decreases and then increases.

and the volume increment from both swelling and growth so that we can indirectly know how luminal area changes. From Sec. 4, we see that the swelling-morphoelasticity model with $r-\theta$ isotropic growth shows almost identical modeling results regardless of how much volume change contribution is from swelling or growth. Such 2-D $r-\theta$ isotropic growth also closely matches the axisymmetric deformation formulation in (3.1). We also use such isotropic growth in this section for convenience of analysis. Figure 5 shows several curves of $r_{i}$ (normalized by $R_{i}$ ) vs. volume increment ratio for several $q$ values, which indicate how volume change alters the shear modulus of the tissue. For each curve, it shows the inner radius first decreases and then increases. This implies that the lumen does not always shrink but can also expand when $r_{i}$ reaches a minimum value. Larger $q$ makes the minimum value larger.

\subsection{Further anticavitation analysis}

We analyze if it is possible for the outer cartilaginous layer to push the inner submucous layer using only finite force such that the inner opening is completely collapsed under TA, which we call anticavitation (Fig. 6). Similar problems have been examined by other researchers. Abeyaratne and Hou studied void collapse for an incompressible spherical elastic solid [33, and Moulton and Goriely studied anticavitation and differential growth in elastic shells by morphoelasticity [34]. The present work takes swelling and growth together to study the possibility of tubular collapse. For this purpose, we only consider the deformation of the inner layer, and the effect of the outer layer is to impose a traction on the inner layer. Denote the traction imposed by the outer layer 


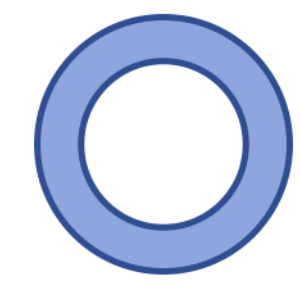

Original inner layer with opening

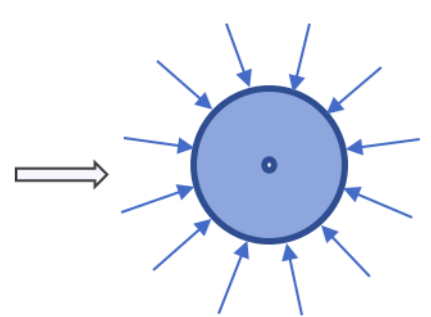

Opening completely closed by pressure from the outer layer

Fig. 6: Anticavitation cartoon for the inner submucous layer with completely closed luminal area under pressure from the outer cartilaginous layer.

through the interface to the inner layer by

$$
-P_{\mathrm{tr}}=\left.T_{r r}^{i}\right|_{R=R_{m}}
$$

where $P_{\text {tr }}>0$.

For any strain energy function $W$ (not only the neo-Hookean model), consider it depends on the three principal stretches $\Lambda_{r}, \Lambda_{\theta}$ and $\Lambda_{z}$ associated with the elastic tensor $\mathbf{F}_{e}$ as $W\left(\Lambda_{r}, \Lambda_{\theta}, \Lambda_{z}\right)$. We remark that usually energy function is considered to depend on the invariants $I_{1}, I_{2}$ and $I_{3}$ etc., and such stretch dependence on the energy function is solely for convenience of the present analysis. Under the theory of swelling-morphoelasticity in Sec. 2.3 and the axisymmetric deformation in 3.1

$$
\mathbf{F}_{e}=\Lambda_{r} \mathbf{e}_{R} \otimes \mathbf{e}_{R}+\Lambda_{\theta} \mathbf{e}_{\Theta} \otimes \mathbf{e}_{\Theta}+\Lambda_{z} \mathbf{e}_{Z} \otimes \mathbf{e}_{Z},
$$

and by virtue of 3.4 ,

$$
\Lambda_{r}=\frac{1}{\alpha}, \quad \Lambda_{\theta}=\frac{\nu g_{z} \alpha}{\lambda_{z}}, \quad \Lambda_{z}=\frac{\lambda_{z}}{g_{z}} .
$$

By (2.14),

$$
\Lambda_{r}=\frac{\nu g_{z}}{\lambda_{z} \Lambda_{\theta}}, \quad \frac{\mathrm{d} \Lambda_{r}}{\mathrm{~d} \Lambda_{\theta}}=-\frac{g_{z} \nu}{\lambda_{z} \Lambda_{\theta}^{2}} .
$$

We consider $P_{\mathrm{tr}}$ as a function of $\Lambda_{\theta}$, and analyze under what condition

$$
\lim _{\Lambda_{\theta}^{i} \rightarrow 0} P_{\operatorname{tr}}<\infty
$$

where $\Lambda_{\theta}^{i}=\left.\Lambda_{\theta}\right|_{r=r_{i}}$.

Generalizing the function $W$ to include swelling with an amount $\nu$ for the inner layer generates

$$
W_{i}=\nu^{q} W\left(\nu^{-1 / 3} \Lambda_{r}, \nu^{-1 / 3} \Lambda_{\theta}, \nu^{-1 / 3} \Lambda_{z}\right) .
$$


Notice that if $W$ is of the neo-Hookean model incorporating the fiber energy, then (5.5) gives (3.10), the generalized neo-Hookean model. Under (2.16),

$$
\begin{aligned}
& T_{r r}^{i}=-p_{i}+\frac{2}{\nu} \Lambda_{r}^{2} \frac{\mathrm{d} W_{i}}{\mathrm{~d} C_{r r}^{e}}, \\
& T_{\theta \theta}^{i}=-p_{i}+\frac{2}{\nu} \Lambda_{\theta}^{2} \frac{\mathrm{d} W_{i}}{\mathrm{~d} C_{\theta \theta}^{e}},
\end{aligned}
$$

where $C_{r r}^{e}$ and $C_{\theta \theta}^{e}$ are the first two principal components of $\mathbf{C}_{\mathbf{e}}$. By the chain rule and the identity $C_{r r}^{e}=\Lambda_{r}^{2}$,

$$
\begin{aligned}
\frac{\mathrm{d} W_{i}}{\mathrm{~d} C_{r r}^{e}} & =\nu^{q} \frac{\partial W}{\partial\left(\nu^{-1 / 3} \Lambda_{r}\right)} \frac{\mathrm{d}\left(\nu^{-1 / 3} \Lambda_{r}\right)}{\mathrm{d} \Lambda_{r}} \frac{\mathrm{d} \Lambda_{r}}{\mathrm{~d} C_{r r}^{e}} \\
& =\frac{\nu^{q-1 / 3}}{2 \Lambda_{r}} \frac{\partial W}{\partial\left(\nu^{-1 / 3} \Lambda_{r}\right)} .
\end{aligned}
$$

Similarly by $C_{\theta \theta}^{e}=\Lambda_{\theta}^{2}$,

$$
\begin{aligned}
\frac{\mathrm{d} W_{i}}{\mathrm{~d} C_{\theta \theta}^{e}} & =\nu^{q} \frac{\partial W}{\partial\left(\nu^{-1 / 3} \Lambda_{\theta}\right)} \frac{\mathrm{d}\left(\nu^{-1 / 3} \Lambda_{\theta}\right)}{\mathrm{d} \Lambda_{\theta}} \frac{\mathrm{d} \Lambda_{\theta}}{\mathrm{d} C_{\theta \theta}^{e}} \\
& =\frac{\nu^{q-1 / 3}}{2 \Lambda_{\theta}} \frac{\partial W}{\partial\left(\nu^{-1 / 3} \Lambda_{\theta}\right)} .
\end{aligned}
$$

By (5.8) and (5.9), Equations (5.6) and (5.7) yield

$$
\begin{aligned}
T_{r r}^{i}-T_{\theta \theta}^{i} & =-\frac{\Lambda_{\theta}}{\nu}\left(-\frac{\partial W}{\partial\left(\nu^{-1 / 3} \Lambda_{r}\right)} \frac{\nu^{q+2 / 3} g_{z}}{\lambda_{z} \Lambda_{\theta}^{2}}+\frac{\partial W}{\partial\left(\nu^{-1 / 3} \Lambda_{\theta}\right)} \nu^{q-1 / 3}\right) \\
& =-\frac{\Lambda_{\theta}}{\nu} \frac{\mathrm{d} W_{i}}{\mathrm{~d} \Lambda_{\theta}} .
\end{aligned}
$$

By the equilibrium equation (3.16) and 5.10 ,

$$
\begin{aligned}
\frac{\mathrm{d} T_{r r}^{i}}{\mathrm{~d} r} & =-\frac{1}{r}\left(T_{r r}^{i}-T_{\theta \theta}^{i}\right) \\
& =\frac{\Lambda_{\theta}}{r \nu} \frac{\mathrm{d} W_{i}}{\mathrm{~d} \Lambda_{\theta}} .
\end{aligned}
$$

Integrating (5.11) with respect to $r$ from $r_{i}$ to $r_{m}$ produces

$$
\int_{r_{i}}^{r_{m}} \frac{\mathrm{d} T_{r r}^{i}}{\mathrm{~d} r} \mathrm{~d} r=\frac{1}{\nu} \int_{r_{i}}^{r_{m}} \frac{\Lambda_{\theta}}{r} \frac{\mathrm{d} W_{i}}{\mathrm{~d} \Lambda_{\theta}} \mathrm{d} r .
$$

Traction free inner boundary $\left.T_{r r}^{i}\right|_{r=r_{i}}=0$ and (5.1) applied to 5.12 give

$$
P_{\mathrm{tr}}=-\frac{1}{\nu} \int_{r_{i}}^{r_{m}} \frac{\Lambda_{\theta}}{r} \frac{\mathrm{d} W_{i}}{\mathrm{~d} \Lambda_{\theta}} \mathrm{d} r
$$


Employing variable change to turn the integration with respect to $\Lambda_{\theta}$ from $\Lambda_{\theta}^{i}=\left.\Lambda_{\theta}\right|_{r=r_{i}}$ to $\Lambda_{\theta}^{m}=\left.\Lambda_{\theta}\right|_{r=r_{m}}$ yields

$$
P_{\mathrm{tr}}=-\frac{1}{\nu} \int_{\Lambda_{\theta}^{i}}^{\Lambda_{\theta}^{m}} \Lambda_{\theta} \frac{\mathrm{d} W_{i}}{\mathrm{~d} \Lambda_{\theta}} \frac{\mathrm{d}(\ln r)}{\mathrm{d} \Lambda_{\theta}} \mathrm{d} \Lambda_{\theta} .
$$

By (3.7) with constant $\nu, g_{r}, g_{\theta}, g_{z}$ and $\lambda_{z}$, we get

$$
r=\left(\frac{\nu g_{r} g_{\theta} g_{z}}{\lambda_{z}}\left(R^{2}-R_{i}^{2}\right)+r_{i}^{2}\right)^{1 / 2} \quad \text { or } \quad R=\left(\frac{\lambda_{z}\left(r^{2}-r_{i}^{2}\right)}{\nu g_{r} g_{\theta} g_{z}}+R_{i}^{2}\right)^{1 / 2} \text {. }
$$

Then by $(3.5)_{2},(5.3)_{2}$ and 5.15 ,

$$
\Lambda_{\theta}=\frac{r}{g_{\theta} R}=r g_{\theta}^{-1}\left(\frac{\lambda_{z}\left(r^{2}-r_{i}^{2}\right)}{\nu g_{r} g_{\theta} g_{z}}+R_{i}^{2}\right)^{-1 / 2} \text {. }
$$

Solving for $r$ from 5.16 generates

$$
r=\Lambda_{\theta}\left(\frac{g_{\theta}\left(\lambda_{z} r_{i}^{2}-R_{i}^{2} \nu g_{r} g_{\theta} g_{z}\right)}{\Lambda_{\theta}^{2} g_{\theta} \lambda_{z}-\nu g_{r} g_{z}}\right)^{1 / 2}
$$

By (5.17),

$$
\frac{\mathrm{d}(\ln r)}{\mathrm{d} \Lambda_{\theta}}=\frac{-\nu g_{r} g_{z}}{\Lambda_{\theta}\left(\Lambda_{\theta} \sqrt{g_{\theta} \lambda_{z}}+\sqrt{\nu g_{r} g_{z}}\right)\left(\Lambda_{\theta} \sqrt{g_{\theta} \lambda_{z}}-\sqrt{\nu g_{r} g_{z}}\right)} .
$$

Plugging 5.18 into 5.14 brings

$$
P_{\mathrm{tr}}=\int_{\Lambda_{\theta}^{i}}^{\Lambda_{\theta}^{m}} \frac{\mathrm{d} W_{i}}{\mathrm{~d} \Lambda_{\theta}} \frac{g_{r} g_{z}}{\left(\Lambda_{\theta} \sqrt{g_{\theta} \lambda_{z}}+\sqrt{\nu g_{r} g_{z}}\right)\left(\Lambda_{\theta} \sqrt{g_{\theta} \lambda_{z}}-\sqrt{\nu g_{r} g_{z}}\right)} \mathrm{d} \Lambda_{\theta} .
$$

Lemma 1 For a $r$ - $\theta$ isotropic (isotropy over the $r-\theta$ plane) one-layered TO under swelling (with the swelling ratio $\nu$ ) and isotropic growth (with three principal growth parameters $g_{r}, g_{\theta}$ and $g_{z}$ satisfying $\left.g_{r}=g_{\theta}\right)$, the following inequality holds

$$
\frac{d W_{i}}{d \Lambda_{\theta}}\left(\Lambda_{\theta} \sqrt{g_{\theta} \lambda_{z}}-\sqrt{\nu g_{r} g_{z}}\right)>0 .
$$

Proof For a planar $r-\theta$ isotropic material under $r-\theta$ isotropic growth and swelling, the Baker-Ericksen inequality [35] holds due to the strong ellipticity of rank one convexity:

$$
\left(T_{r r}^{i}-T_{\theta \theta}^{i}\right)\left(\lambda_{r}-\lambda_{\theta}\right)>0,
$$

where $\lambda_{r}$ and $\lambda_{\theta}$ are two principal stretches of $\mathbf{F}$ in the $r$ and $\theta$ directions, respectively, satisfying $\lambda_{r}=\Lambda_{r} g_{r}$ and $\lambda_{\theta}=\Lambda_{\theta} g_{\theta}$. By the definition of $\Lambda_{r}$ and $\Lambda_{\theta}$ in (5.3) and Eq. (3.4), one has $\alpha=\frac{\Lambda_{\theta} \lambda_{z}}{\nu g_{z}}$. Accordingly,

$$
\begin{aligned}
\lambda_{r}-\lambda_{\theta} & =\frac{g_{r}}{\alpha}-\frac{\nu g_{z} \alpha g_{\theta}}{\lambda_{z}} \\
& =\frac{\left(\sqrt{g_{r} g_{z} \nu}-\Lambda_{\theta} \sqrt{g_{\theta} \lambda_{z}}\right)\left(\sqrt{g_{r} g_{z} \nu}+\Lambda_{\theta} \sqrt{g_{\theta} \lambda_{z}}\right)}{\Lambda_{\theta} \lambda_{z}} .
\end{aligned}
$$

By 5.10 for $T_{r r}^{i}-T_{\theta \theta}^{i}$ and Eq. 5.22, one thus obtains 5.20 for the lemma. 
Lemma 2 For $\Lambda_{\theta}^{i} \leq \Lambda_{\theta} \leq \Lambda_{\theta}^{m}, \Lambda_{\theta} \sqrt{g_{\theta} \lambda_{z}}-\sqrt{\nu g_{r} g_{z}}<0$ as $r_{i}$ approaches 0 and is small enough.

Proof By 5.3$)_{2}$ and $(3.5)_{1}$,

$$
\begin{aligned}
\Lambda_{\theta} \sqrt{g_{\theta} \lambda_{z}}-\sqrt{\nu g_{r} g_{z}} & =\sqrt{g_{\theta} \lambda_{z}} \frac{\nu g_{z}}{\lambda_{z}} \alpha-\sqrt{\nu g_{r} g_{z}} \\
& =\sqrt{g_{\theta} \lambda} \frac{\nu g_{z}}{\lambda_{z}} g_{r}\left(\frac{\mathrm{d} r}{\mathrm{~d} R}\right)^{-1}-\sqrt{\nu g_{r} g_{z}} \\
& =\frac{\sqrt{\lambda_{z}} r}{\sqrt{g_{\theta}} R}-\sqrt{\nu g_{r} g_{z}} .
\end{aligned}
$$

By the volume relation between the reference configuration and the deformed configuration,

$$
\left(R_{m}^{2}-R_{i}^{2}\right) \nu g_{r} g_{\theta} g_{z}=\left(r_{m}^{2}-r_{i}^{2}\right) \lambda_{z}
$$

we can obtain

$$
r_{m}=\sqrt{\frac{\left(R_{m}^{2}-R_{i}^{2}\right) \nu g_{r} g_{\theta} g_{z}}{\lambda_{z}}+r_{i}^{2}}
$$

By 5.23 and 5.25 ,

$$
\left.\lim _{r_{i} \rightarrow 0}\left(\Lambda_{\theta} \sqrt{g_{\theta} \lambda_{z}}-\sqrt{\nu g_{r} g_{z}}\right)\right|_{r=r_{m}}=\sqrt{\nu g_{r} g_{z}}\left(\sqrt{1-\frac{R_{i}^{2}}{R_{m}^{2}}}-1\right)<0 .
$$

Therefore, as $r_{i} \rightarrow 0$ and is small enough, $\Lambda_{\theta} \sqrt{g_{\theta} \lambda_{z}}-\sqrt{\nu g_{r} g_{z}}<0$ for all $\Lambda_{\theta}^{i} \leq \Lambda_{\theta} \leq \Lambda_{\theta}^{m}$.

By Lemmas 1 and 2 applied on (5.19), one has

$$
\begin{aligned}
0<P_{\mathrm{tr}} & <\sqrt{\frac{g_{r} g_{z}}{\nu}} \int_{\Lambda_{\theta}^{i}}^{\Lambda_{\theta}^{m}} \frac{\mathrm{d} W_{i}}{\mathrm{~d} \Lambda_{\theta}} \frac{\mathrm{d} \Lambda_{\theta}}{\Lambda_{\theta} \sqrt{g_{\theta} \lambda_{z}}-\sqrt{\nu g_{r} g_{z}}} \\
& <\sqrt{\frac{g_{r} g_{z}}{\nu}} \frac{1}{\Lambda_{\theta}^{m} \sqrt{g_{\theta} \lambda_{z}}-\sqrt{\nu g_{r} g_{z}}} \int_{\Lambda_{\theta}^{i}}^{\Lambda_{\theta}^{m}} \frac{\mathrm{d} W_{i}}{\mathrm{~d} \Lambda_{\theta}} \mathrm{d} \Lambda_{\theta} \\
& =\sqrt{\frac{g_{r} g_{z}}{\nu}} \frac{1}{\Lambda_{\theta}^{m} \sqrt{g_{\theta} \lambda_{z}}-\sqrt{\nu g_{r} g_{z}}}\left(\left.W_{i}\right|_{\Lambda_{\theta}=\Lambda_{\theta}^{m}}-\left.W_{i}\right|_{\Lambda_{\theta}=\Lambda_{\theta}^{i}}\right) \\
& =K\left(\left.W_{i}\right|_{\Lambda_{\theta}=\Lambda_{\theta}^{i}}-\left.W_{i}\right|_{\Lambda_{\theta}=\Lambda_{\theta}^{m}}\right),
\end{aligned}
$$

where $K=\sqrt{\frac{g_{r} g_{z}}{\nu}} \frac{1}{\sqrt{\nu g_{r} g_{z}}-\Lambda_{\theta}^{m} \sqrt{g_{\theta} \lambda_{z}}}>0$.

Theorem 3 The inner submucous layer can be completely collapsed under finite pressure from the outer cartilaginous layer if and only if the following is true

$$
\left.\lim _{\Lambda_{\theta}^{i} \rightarrow 0} W_{i}\right|_{\Lambda_{\theta}=\Lambda_{\theta}^{i}}=\mathbb{L}<\infty,
$$

where $\mathbb{L}$ is any positive finite number. 
Proof If (5.28) holds, then by (5.27), $\left.P_{\mathrm{tr}}\right|_{\Lambda_{\theta}^{i}=0}$ is bounded, and finite pressure is possible to totally collapse the lumen of the trachea.

Instead, if $\left.P_{\operatorname{tr}}\right|_{\Lambda_{\theta}^{i}=0}$ is bounded, we show 5.28 is true. By Lemma 2

$$
0>-\frac{1}{\sqrt{\nu g_{r} g_{z}}}>\frac{1}{\Lambda_{\theta} \sqrt{g_{\theta} \lambda_{z}}-\sqrt{\nu g_{r} g_{z}}},
$$

for $\Lambda_{\theta}^{i} \leq \Lambda_{\theta} \leq \Lambda_{\theta}^{m}$. Thus by 5.19

$$
\begin{aligned}
P_{\mathrm{tr}} & >-\frac{1}{\sqrt{\nu g_{r} g_{z}}} \int_{\Lambda_{\theta}^{i}}^{\Lambda_{\theta}^{m}} \frac{\mathrm{d} W_{i}}{\mathrm{~d} \Lambda_{\theta}} \frac{g_{r} g_{z}}{\left(\Lambda_{\theta} \sqrt{g_{\theta} \lambda_{z}}+\sqrt{\nu g_{r} g_{z}}\right)} \mathrm{d} \Lambda_{\theta} \\
& >\frac{-\sqrt{g_{r} g_{z}}}{\sqrt{\nu}\left(\Lambda_{\theta}^{m} \sqrt{g_{\theta} \lambda_{z}}+\sqrt{\nu g_{r} g_{z}}\right)} \int_{\Lambda_{\theta}^{i}}^{\Lambda_{\theta}^{m}} \frac{\mathrm{d} W_{i}}{\mathrm{~d} \Lambda_{\theta}} \mathrm{d} \Lambda_{\theta} \\
& =\frac{\sqrt{g_{r} g_{z}}}{\sqrt{\nu}\left(\Lambda_{\theta}^{m} \sqrt{g_{\theta} \lambda_{z}}+\sqrt{\nu g_{r} g_{z}}\right)}\left(\left.W_{i}\right|_{\Lambda_{\theta}=\Lambda_{\theta}^{i}}-\left.W_{i}\right|_{\Lambda_{\theta}^{m}}\right),
\end{aligned}
$$

where we also used $\frac{\mathrm{d} W_{i}}{\mathrm{~d} \Lambda_{\theta}}<0$ obtained from Lemmas 1 and 2 . Since $W_{i}$ as an energy function is always non-negative and $\lim _{\Lambda^{i}} P_{\mathrm{tr}}$ is bounded, we get

$$
\infty>\left.\lim _{\Lambda_{\theta}^{i} \rightarrow 0} W_{i}\right|_{\Lambda_{\theta}=\Lambda_{\theta}^{i}} \geq 0
$$

which gives (5.28).

Corollary 1 For the generalized neo-Hookean model (2.7) incorporating swelling, the anticavitation of the inner submucous layer can not occur under finite pressure from the outer cartilaginous layer.

Proof By (5.2) and (5.3), $I_{1}=\operatorname{tr} \mathbf{C}_{e}=\Lambda_{r}^{2}+\Lambda_{\theta}^{2}+\Lambda_{z}^{2}$ and $I_{4}=\Lambda_{z}^{2}$ (fiber direction parallel to the $\mathbf{e}_{z}$ direction). And then 2.14 gives $\Lambda_{r}=\frac{\nu}{\Lambda_{\theta} \Lambda_{z}}$. The generalized neo-Hookean model 3.10 becomes

$$
W_{i}=\frac{\mu_{i} \nu^{q-2 / 3}}{2}\left(\left(\frac{\nu}{\Lambda_{\theta} \Lambda_{z}}\right)^{2}+\Lambda_{\theta}^{2}+\Lambda_{z}^{2}-3 \nu^{2 / 3}\right)+\frac{\gamma}{2}\left(\Lambda_{z}^{2}-1\right)^{2}
$$

which easily shows that

$$
\left.\lim _{\Lambda_{\theta}^{i} \rightarrow 0} W_{i}\right|_{\Lambda_{\theta}=\Lambda_{\theta}^{i}}=\infty
$$

Therefore, by Theorem 3 the present generalized neo-Hookean model is incapable of closing the inner channel through finite pressure imposed on the inner submucous layer by the outer cartilaginous layer.

The neo-Hookean model coefficient $\frac{\mu_{i} \nu^{q-2 / 3}}{2}$ in 3.10 under the limit $q \rightarrow$ $-\infty$ means that the shear modulus of the tissue is annihilated due to swelling, and thus

$$
\lim _{\Lambda_{\theta}^{i} \rightarrow 0}\left(\left.W_{i}\right|_{q \rightarrow-\infty, \Lambda_{\theta}=\Lambda_{\theta}^{i}}\right)=0 .
$$

So by Theorem 3 the total collapse is possible with finite pressure from the outer layer under this extreme case. 


\section{Summary and discussion}

The trachea is an important human tubular organ. When angioedema occurs in this organ, the normal size of the inner lumen may be changed, which can cause serious breathing difficulty. Trachea is idealized to be a two-layered cylinder. Angioedema is taken to occur in the inner softer layer, and the outer cartilaginous layer is modeled as being incompressible. Inner and outer boundaries of the trachea is taken to be traction free to neglect the canal air flow and outer skin influence over the trachea configuration.

TA is modeled employing a new nonlinear swelling-morphoelasticity theory formed based on the swelling theory and morphoelasticity theory. Both theories are generalized hyperelasticity to model volume change behavior of a continuum body. However, they are designed from different perspectives. For the first one, the deformation gradient from the undeformed to the final deformed configuration is used in the updated strain energy functions to derive the stress distribution, and volume change is used to constrain the determinant of the deformation gradient tensor. For the second one, such deformation gradient is decomposed to be a growth tensor and an elastic tensor, and only the elastic tensor is employed to generated the stress distribution. The volume change is included in the growth tensor part. The newly created swellingmorphoelasticity theory combines the two theories in one model, and is more flexible to model deformation under volume change by distributing it between the swelling part and the growth part according to different physiological results. Such theory can also be employed in compressible models for convenience of finite-element generation of the computational results based on [27]. This new theory is advantageous to model TA, because its volume change is also from such two parts with the swelling part caused by fluid leakage from blood vessels and the solid growth part caused by mass addition to the tracheal tissue [11.

Computational results are given for the deformed radius $r$ vs. the undeformed radius $R$, and the three principal Cauchy stress components $T_{r r}, T_{\theta \theta}$, and $T_{z z}$ vs. $R$. The results are reflective of the prescribed boundary conditions, and show nonlinear variation through the domain. More particularly, the graphs of $T_{r r}$ vs. $R$ show residual stress concentration near the interface of the two layers. Graphs for $T_{\theta \theta}$ vs. $R$ and $T_{z z}$ vs. $R$ are discontinuous through the interface, being compressive in the inner layer and tensile in the outer layer. Theories of morphoelasticity and swelling are structurally different, but it is found that the computational results for the swelling theory, morphoelasticity theory and their mix are qualitatively similar to each other with slight quantitative difference. Though swelling theory is three dimensional in default, the results from 3-D isotropic growth for morphoelasticity do not match those from the swelling theory. Instead the results from 2-D $r-\theta$ isotropic growth of morphoelasticity is nearly identical to those from swelling theory, regardless of how the volume change contribution is from growth or swelling. A summary of the computational results in Sec. 4 is demonstrated in Table 3 . A further 


\begin{tabular}{|ccccc|}
\hline & $r$ & $\left|T_{r r}\right|$ & $\left|T_{\theta \theta}\right|$ & $\left|T_{z z}\right|$ \\
\hline 3-D i.g.: & Growth dom. & Growth dom. & Growth dom. & Growth dom. \\
\hline$r-\theta$ i.g.: & The same & The same & The same & The same over $\left[R_{m}, R_{o}\right]$ \\
\hline$r-z$ i.g.: & Swelling dom. & Swelling dom. & Swelling dom. & Swelling dom. \\
\hline$\theta-z$ i.g.: & Growth dom. & Growth dom. & Growth dom. & Growth dom. \\
\hline
\end{tabular}

Table 3: Summary of computational results comparison for the swelling-morphoelasticity model. The same amount of volume increment caused by a combination of swelling and growth is used for all the comparisons. Here "i.g." stands for isotropic growth, and "dom." is the abbreviation of "dominate". The symbol $|\bullet|$ means the absolute value of the quantity inside. For the row of 3-D isotropic growth, "Growth dom." means more growth and less swelling generate larger material radius change and magnitude of $T_{r r}, T_{\theta \theta}$, and $T_{z z}$ under the same $R$ location. So does the row for $\theta-z$ isotropic growth. For the $r-z$ isotropic growth, the trend is the opposite. For the $r-\theta$ isotropic growth row, the results are always the same (except $T_{z z}$ ) regardless of how combination of swelling and growth contribution to the volume increment is made.

theoretical exploration is needed to identify the connection of swelling theory and morphoelasticity theory based on these results.

Non-monotonic inner boundary radius change is also shown. The deformed inner radius $r_{i}$ decreases as inner layer volume becomes large in the beginning, but after the volume increment reaches some point depending on how such increment affects the stiffness, $r_{i}$ unexpectedly increases generating a larger lumen. This nonlinear characteristics is also exhibited in other TOs. For example, Glagov et al. 32] experimentally discovered that the coronary arteries with atherosclerotic plaques along the inner arterial wall started to increase their luminal area in the beginning, and later as the stenosis grew larger, the luminal area decreased. Fok 30 employed the morphoelasticity theory to model such plaque growth and successfully captured similar phenomena. He also found that such nonlinear growth behavior also depended on in vivo or ex vivo environment of the vessels. The nonlinearity patterns of the inner radius change for the trachea and arteries are distinct, which may be caused by other detailed structural difference of the two organs.

To further explore the nonlinear inner radius change and check possibility of complete lumen closure, an anticavitation analysis is made based on general strain energy density functions for the swelling-morphoelasticity theory. The theoretical analysis is based on the inner layer, where the outer layer imposes an inward pressure on the boundary of it. We rigorously showed that the lumen can be completely closed with finite force from the outer layer if and only if the strain energy remains finite as the angular stretch goes to zero. The commonly used neo-Hookean model does not satisfy this requirement. Therefore in angioedema, it is theoretically impossible to completely close off a neo-Hookean trachea no matter how much growth or swelling occurs.

When under volume increment from either growth or swelling, the inner wall of the soft tissues may demonstrate asymmetric buckling behaviors [2,3, 38,44 . One can study these instabilities for an axisymmetric base state, and finite element approaches need to be employed for more general geometries. 
Even in the axisymmetric setting, it would be interesting to see how the classical buckling analysis changes under our new swelling-morphoelasticity theory.

Although the present work focuses on a particular organ (the trachea), our results are indicative of multi-layer TOs modeled using hyperelasticity. Because different layers have different stiffness parameters, thickness or fiber family embedding, the computed size change and stress distribution may be non-smooth, discontinuous, or non-monotonic. Stresses may concentrate near the interface between layers, making the tissue there more vulnerable to damage or dislocation. We predict that TOs will not close their lumens completely when their inner layers experience growth and/or swelling. These nonlinear results can assist us to understand other TO deformation behaviors for corresponding physiological problems.

\section{References}

1. Beitel GJ, Krasnow MA (2000) Genetic control of epithelial tube size in the Drosophila tracheal system. Dev 127:3271-3282

2. Ciarletta P, Balbi V, Kuhl E (2014) Pattern selection in growing tubular tissues. Phys Rev Lett 113:248101:1-5

3. Ciarletta P, Amar MB (2012) Pattern formation in fiber-reinforced tubular tissues: folding and segmentation during epithelial growth. J Mech Phys Solids 60(3):525-537

4. Gregersen H, Barlow J, Thompson D (1999) Development of a computer-controlled tensiometer for real-time measurements of tension in tubular organs. Neurogastroenterol 11:109-118

5. Minnich DJ, Mathisen DJ (2007) Anatomy of the trachea, carina, and bronchi. Thorac Surg Clin 17(4):571-585

6. Gu WY, Lai WM, Mow VC (1998) A mixture theory for charged-hydrated soft tissues containing multi-electrolytes: passive transport and swelling behaviors. J Biomech Eng 120(2):169-180

7. Singh PP, Cushman JH, Maier DE (2003) Multiscale fluid transport theory for swelling biopolymers. Chem Eng Sci 58(11):2409-2419

8. Baek S, Pence TJ (2011) Inhomogeneous deformation of elastomer gels in equilibrium under saturated and unsaturated conditions. J Mech Phys Solids 59:561-582

9. Wang L, Tepper R, Bert JL, Pinder KL, Par PD, Okazawa M (2000) Mechanical properties of the tracheal mucosal membrane in the rabbit. I. Steady-state stiffness as a function of age. J Appl Physiol 88:1014-1021

10. Katz MA, Bresler EH (1984) Osmosis. In: Edema, p 39-46

11. Temio VM, Peebles RS (2008) The spectrum and treatment of angioedema. Am J Med 121:282-286

12. Greaves M, Lawlor F (1991) Angioedema: manifestations and management. J Am Acad Dermatology 25:155-165

13. Natali AN, Carniel EL, Pavan PG, Dario P, Izzo I (2006) Hyperelastic models for the analysis of soft tissue mechanics: definition of constitutive parameters. In: The First IEEE/RAS-EMBS International Conference on Biomedical Robotics and Biomechatronics, p 1-8

14. Bork K (2006) Hereditary angioedema with normal c1 inhibitor activity including hereditary angioedema with coagulation factor xii gene mutations. Immunol Allergy Clin North Am 26:709-724

15. Krnacik MJ, Heggeness MH (1997) Severe angioedema causing airway obstruction after anterior cervical surgery. Spine 22:2188-2190

16. Ishoo E, Shah UK, Grillone GA, Stram JR, Fuleihan NS (1999) Predicting airway risk in angioedema: staging system based on presentation. Otolaryngol Head Neck 121:263-268 
17. Bork K, Hardt J, Schicketanz KH, Ressel N (2003) Clinical studies of sudden upper airway obstruction in patients with hereditary angioedema due to C1 esterase inhibitor deficiency. Arch Intern Med 163:1229-1235

18. Brown RH, Zerhouni EA, Mitzner W (1995) Airway edema potentiates airway reactivity. J Appl Physiol 79:1242-1248

19. Amin SD, Majumdar A, Frey U, Suki B (2010) Modeling the dynamics of airway constriction: effects of agonist transport and binding. J Appl Physiol 109:553-563

20. Holzapfel GA (2001) Biomechanics of soft tissue. In: The handbook of materials behavior, $\mathrm{p}$ 1-12

21. Aukland K, Reed RK (1993) Interstitial-lymphatic mechanisms in the control of extracellular fluid volume. Physiol Rev 73:1-78

22. Reed RK (1979) An implantable colloid osmometer: Measurements in subcutis and skeletal muscle of rats. Microvasc Res 18:83-94

23. Bennethum LS, Cushman JH (1996) Multiscale, hybrid mixture theory for swelling systems-I: balance laws. Int J Eng Sci 34:125-145

24. Gou K, Pence TJ (2016) Hyperelastic modeling of swelling in fibrous soft tissue with application to tracheal angioedema. J Math Biol 72:499-526

25. Rodriguez EK, Hoger A, McCulloch AD (1994) Stress-dependent finite growth in soft elastic tissues. J Biomech 27:455-467

26. Goriely A, Moulton D (2011) Morphoelasticity: a theory of elastic growth. Oxford University Press

27. Gou K, Pen TJ (2015) On compressible versions of the incompressible neo-Hookean material. Math Mech Solids 20:157-182

28. Demirkoparana H, Pence TJ (2007) Swelling of an internally pressurized nonlinearly elastic tube with fiber reinforcing. Int J Solids Struct 44:4009-4029

29. Gou K, Pence TJ (2017) Computational modeling of tracheal angioedema due to swelling of the submucous tissue layer. Int $\mathrm{J}$ Numer Method Biomed Eng. DOI: $10.1002 / \mathrm{cnm} .2861$

30. Fok PW (2016) Multi-Layer mechanical model of Glagov remodeling in coronary arteries: Differences between In-Vivo and Ex-Vivo Measurements. PloS one 11: e0159304

31. Fok PW, Sanft R (2015) A biochemical and mechanical model of injury-induced intimal thickening. Math Med Biol 34:77-108

32. Glagov S, Weisenberg E, Zarins CK, Stankunavicius R, Kolettis GJ (1987) Compensatory enlargement of human atherosclerotic coronary arteries. N Engl J Med 316:13711375

33. Abeyaratne R, Hou HS (1991) Void collapse in an elastic solid. J Elast 26:23-42

34. Moulton DE, Goriely A (2011) Anticavitation and differential growth in elastic shells. J Elast 102:117-132

35. Fosdick R, Silhavy M (2006) Generalized Baker-Ericksen inequalities. J Elast 85:39-44

36. Tsai H, Pence TJ (2004) Swelling induced finite strain flexure in a rectangular block of an isotropic elastic material. J Elast 75:69-89

37. Pence TJ, Tsai H (2006) Swelling-induced cavitation of elastic spheres. Math Mech Solids 11:527-551

38. Demirkoparan H (2017) Bulging bifurcation of inflated circular cylinders of doubly fiber-reinforced hyperelastic material under axial loading and swelling. Math Mech Solids 22(4):666-682

39. Demirkoparan H, Merodio J (2017) Swelling-twist interaction in fiber-reinforced hyperelastic materials: the example of azimuthal shear. J Eng Math. DOI 10.1007/s10665-0179906-x

40. Goriely A, Moulton DE, Vandiver R (2010) Elastic cavitation, tube hollowing, and differential growth in plants and biological tissues. Europ Lett 91:18001:1-6

41. Balbi V, Ciarletta P (2013) Morpho-elasticity of intestinal villi. J R Soc Interface 10:20130109:1-12

42. Goriely A, Amar MB (2007) On the definition and modeling of incremental, cumulative, and continuous growth laws in morphoelasticity. Biomech Model Mechanobiol 6:289-296

43. Bowden LG, Byrne HM, Maini PK (2016) A morphoelastic model for dermal wound closure. Biomech Model Mechanobiol 15:663-681

44. Li B, Cao YP, Feng XQ, Gao H (2012) Mechanics of morphological instabilities and surface wrinkling in soft materials: a review. Soft Matter 8:5728-5745 